\title{
Microscope-Cockpit: Python-based bespoke microscopy for
}

\section{bio-medical science [version 1; peer review: 1 approved, 1}

\section{approved with reservations]}

\author{
Mick A. Phillips (D1-3, David Miguel Susano Pinto (Di)1, Nicholas Hall(D1, \\ Julio Mateos-Langerak (D) 4, Richard M. Parton (D1) Josh Titlow', \\ Danail V. Stoychev (iD), Thomas Parks², Tiago Susano Pinto1, John W. Sedat5, \\ Martin J. Booth (iD), Ilan Davis'1, Ian M. Dobbie (iD) \footnotetext{
2Diamond Light Source, Harwell Science and Innovation Campus, Didcot, OX11 ODE, UK

${ }^{3}$ Division of Structural Biology, The Henry Wellcome Building for Genomic Medicine,, Roosevelt Drive, Oxford, OX3 7BN, UK

${ }^{4} \mathrm{IGH}$, Univ Montpellier, Montpellier, 34396, France

${ }^{5}$ Department of Biochemistry \& Biophysics, UCSF, San Francisco, CA, 94158, USA

${ }^{6}$ Department of Engineering Science, University of Oxford, Oxford, OX1 3PJ, UK
} \\ ${ }^{1}$ Micron Advanced Bioimaging Unit, Department of Biochemistry, University of Oxford, South Parks Road, Oxford, OX1 3QU, UK
}

V1 First published: 08 Apr 2021, 6:76

https://doi.org/10.12688/wellcomeopenres.16610.1

Latest published: 17 Jan 2022, 6:76

https://doi.org/10.12688/wellcomeopenres.16610.2

\section{Abstract}

We have developed "Microscope-Cockpit" (Cockpit), a highly adaptable open source user-friendly Python-based Graphical User Interface (GUI) environment for precision control of both simple and elaborate bespoke microscope systems. The user environment allows nextgeneration near instantaneous navigation of the entire slide landscape for efficient selection of specimens of interest and automated acquisition without the use of eyepieces. Cockpit uses "Python-Microscope" (Microscope) for high-performance coordinated control of a wide range of hardware devices using open source software. Microscope also controls complex hardware devices such as deformable mirrors for aberration correction and spatial light modulators for structured illumination via abstracted device models. We demonstrate the advantages of the Cockpit platform using several bespoke microscopes, including a simple widefield system and a complex system with adaptive optics and structured illumination. A key strength of Cockpit is its use of Python, which means that any microscope built with Cockpit is ready for future customisation by simply adding new libraries, for example machine learning algorithms to enable automated microscopy decision making while imaging.

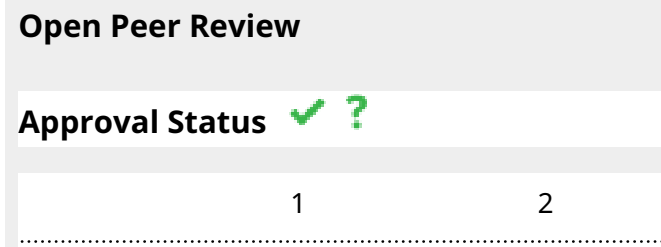

version 2

(revision)

17 Jan 2022

version 1

08 Apr 2021

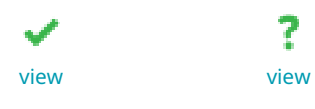

1. Kyle Harrington (iD), Max Delbrueck Center for Molecular Medicine, Berlin, Germany

2. Jason R Swedlow (iD), University of Dundee, Dundee, UK

Emil Rozbicki, Glencoe Software, Inc, Seattle, USA

William Moore, University of Dundee,

Dundee, UK

Sébastien Besson (D), University of Dundee,

Dundee, UK 


\section{Keywords}

Microscope-Python, Bespoke microscope, Microscope hardware device control, Free and open source software, Imaging, Machine Learning (ML), Artificial Intelligence (AI), Adaptive optics (AO), Super resolution microscopy
Any reports and responses or comments on the article can be found at the end of the article.

Corresponding authors: Ilan Davis (ilan.davis@bioch.ox.ac.uk), Ian M. Dobbie (ian.dobbie@jhu.edu)

Author roles: Phillips MA: Conceptualization, Software; Susano Pinto DM: Conceptualization, Software, Supervision, Writing - Original Draft Preparation, Writing - Review \& Editing; Hall N: Software; Mateos-Langerak J: Software; Parton RM: Conceptualization,

Resources; Titlow J: Resources; Stoychev DV: Software; Parks T: Software; Susano Pinto T: Software; Sedat JW: Conceptualization; Booth MJ: Conceptualization, Supervision; Davis I: Conceptualization, Funding Acquisition, Project Administration, Supervision, Writing Original Draft Preparation, Writing - Review \& Editing; Dobbie IM: Conceptualization, Funding Acquisition, Project Administration, Software, Supervision, Writing - Original Draft Preparation, Writing - Review \& Editing

Competing interests: Martin Booth declares a significant interest in Aurox Ltd., whose microscopes were used in this work.

Grant information: This research was funded by Wellcome strategic awards, a senior fellowship and an investigator award to I.D. [091911/Z/10/Z], [091911/Z/10/A], [107457/Z/15/Z], [096144/Z/17/Z]and [209412/Z/17/Z]; Wellcome funding to the Wellcome Trust Centre for Human Genetics [105605/Z/14/Z] and [203141/Z/16/Z]; an MRC/EPSRC/BBSRC Next-generation Optical Microscopy [MR/K01577X/1] to I.D.; GIS IBiSA [\#2015-28]; and by the CNRSMITI [Défi Imag'In 2015]. D.S. is funded by a BBSRC iCASE grant with Aurox as the industrial partner [BB/M011224/1]. N.H. was supported by funding from the Engineering and Physical Sciences Research Council (EPSRC) and Medical Research Council (MRC)[EP/L016052/1] The funders had no role in study design, data collection and analysis, decision to publish, or preparation of the manuscript.

Copyright: (c) 2021 Phillips MA et al. This is an open access article distributed under the terms of the Creative Commons Attribution License, which permits unrestricted use, distribution, and reproduction in any medium, provided the original work is properly cited.

How to cite this article: Phillips MA, Susano Pinto DM, Hall N et al. Microscope-Cockpit: Python-based bespoke microscopy for biomedical science [version 1; peer review: 1 approved, 1 approved with reservations] Wellcome Open Research $2021,6: 76$ https://doi.org/10.12688/wellcomeopenres.16610.1

First published: 08 Apr 2021, 6:76 https://doi.org/10.12688/wellcomeopenres.16610.1 


\section{Introduction}

\section{Why we need Cockpit}

Biomedical research has benefited from significant engineering advances that have increased the speed and sensitivity of many types of scientific instrumentation. Microscope technology, in particular, is evolving rapidly through advances in optical techniques and image processing, enabling major scientific discovery. However, the rapid adoption and application of advanced microscopies by biomedical scientists is constrained, especially when it relies on commercialisation to make the necessary technology accessible to the end-users.

It can often take several years to develop innovations from research instruments into user-friendly, off-the-shelf systems ${ }^{1-4}$. We report a collaboration between the University of Oxford and the University of California San Francisco (UCSF) that grew out of the OMX microscope project, dating from the early 1990s, which is extensively documented in the supplement of 1 .

To accelerate technology adoption, many labs are building custom microscopes based on newly developed imaging methods, such as light sheet ${ }^{5}$, lattice light sheet ${ }^{3}, 3 \mathrm{DSIM}^{1,6}, \mathrm{STED}^{7}$, and MINFLUX $^{8}$, or to exploit new analysis techniques, such as STORM analysis ${ }^{9,10}, 3 \mathrm{~B}^{11}$, and SIMFLUX ${ }^{12}$. These instruments require flexible, computerised control of a wide range of individual components (lasers, mirror actuators, filters, objectives, detectors, etc.) allowing more rapid deployment of novel techniques, and often providing faster and more sensitive operation than commercial systems $\mathrm{s}^{3,13,14}$. However, integration of these components into a single user-friendly platform that is focused on users' scientific application, rather than dictated by the control infrastructure, can be a major challenge. There have been three general solutions adopted by the community for software control of bespoke microscope hardware.

1. Using individual manufacturer-provided packages for each piece of hardware. This can provide a straightforward solution for simpler systems, but fundamental incompatibilities between each vendor's control models can prevent integration of multiple components.

2. Using LabVIEW, a commercial visual programming tool from a leading hardware manufacturer, National Instruments (Austin, Texas, United States).

3. Use of a dedicated microscope control package, of which several are available, with the most widely used being Micromanager ( $\mu$ Manager): an open source Java and $\mathrm{C}++$ based software platform for controlling a range of microscope hardware such as stands, cameras, and stages. A less widely-adopted solution is a bespoke platform written in Python to control the OMX microscope ${ }^{1,15,16}$, and this forms the starting point of the Microscope-Cockpit project described in this manuscript.

An important feature for custom microscopes is the freedom to design the system around a specific set of experiments, as defined by the user. With a specific application in mind, an instrument can be optimised to make the best possible compromises for that application. For example, imaging deep into biological tissue can involve significant optical aberrations leading to degraded image quality. This can be compensated for by the application of adaptive optics techniques, at a cost of increased complexity and marginally decreased light efficiency. However, it is desirable that this capability is easily enabled and exploited by microscope users, typically biologists with limited experience of the underlying engineering, and be reproducible over months or years of use. Many such custom microscope designs have been published ${ }^{17-22}$. In all of these cases, control of the many hardware components in a complex and timed manner is challenging, particularly for time sensitive applications involving living specimens. Correction settings must be measured and applied in coordination while simultaneously minimising additional exposure of the sample.

Finally, the democratisation of artificial intelligence (AI)-based image analysis approaches means that a wealth of tools are potentially available to users. However, current systems, with some specific exceptions, are not well suited for the integration of bespoke novel AI-approaches with the imaging process. This limitation can be significantly reduced by an appropriately flexible package for control of bespoke systems.

\section{Philosophy and implementation of Cockpit}

We set out to create a package that is suitable for biologists to carry out a range of experiments in a time efficient manner, at scale. At the same time, the software has to control a wide range of electronic and optical hardware devices and microscope types. Many bespoke microscope systems that lack eyepieces, such as systems built by physical scientists in collaboration with biologists, make sample navigation particularly challenging. Improving navigation on this type of system is a major feature of Cockpit.

There are several existing software control approaches including manufacturer-supplied packages, custom control software, in LabVIEW or a more traditional programming language, or the open source program $\mu$ Manager, as discussed earlier. We feel that Cockpit has several distinct advantages over these other approaches. We compare the relative merits of Cockpit and a range of alternatives in the discussion.

Cockpit is an open-source software package that makes it easy to control multiple devices through a single Python-based user interface (Figure 1). Cockpit supports multiple platforms, and the similarity of its interface across operating systems and widget toolkits is shown in Figure 2. It provides an innovative mosaic tool, allowing large areas of a microscope specimen to be imaged and then navigated in real time. This is similar to Micro-Magellan ${ }^{23}$, but with the additional feature of keeping even thousands of images in Graphic Processing Unit (GPU) memory for instant access at various levels of detail. It utilises Python-Microscope to communicate with a wide range of microscope hardware and is able to exploit a master timing control device to provide hardware triggering for digital devices and synchronised analogue voltages. This enables highly reproducible timing over a range of time scales even in extremely complex experiments with multiple devices. 


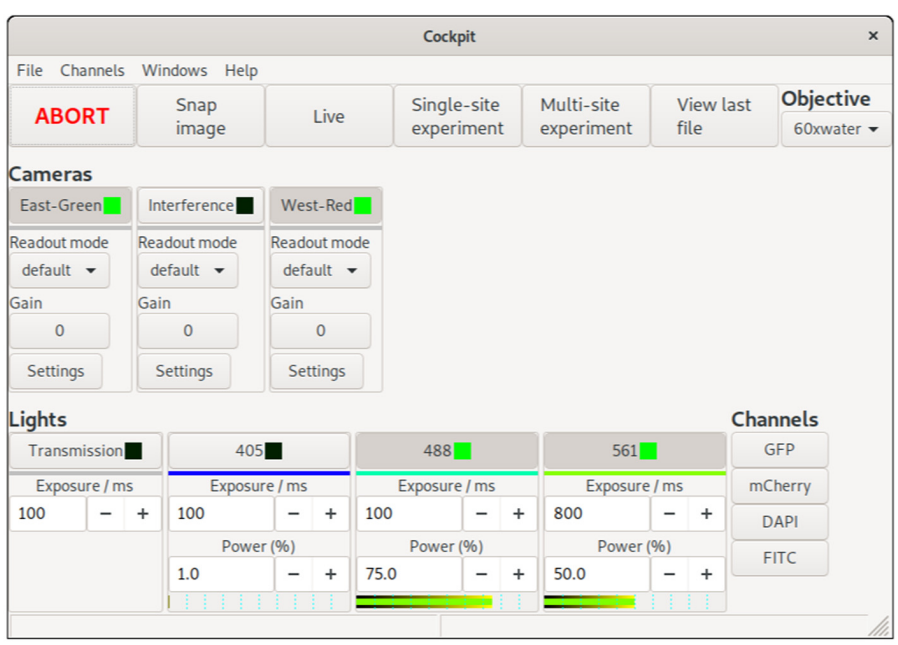

(a) Main Window

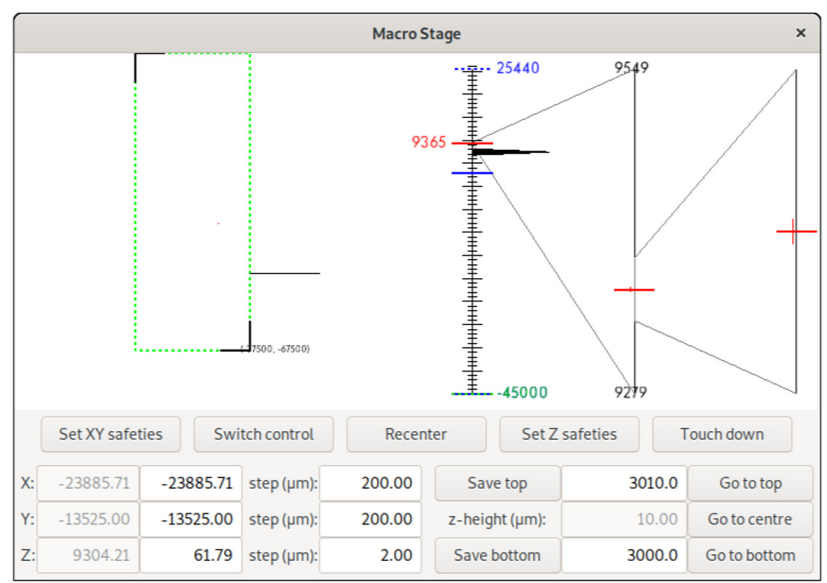

(b) Macro Stage

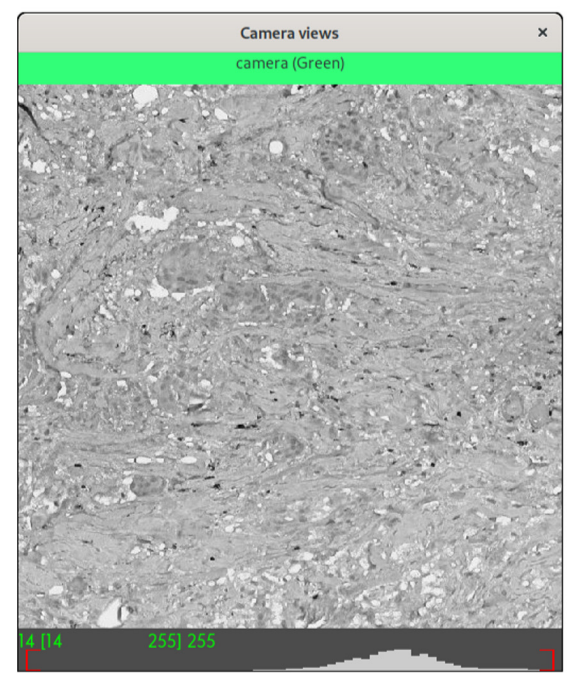

(c) Camera View

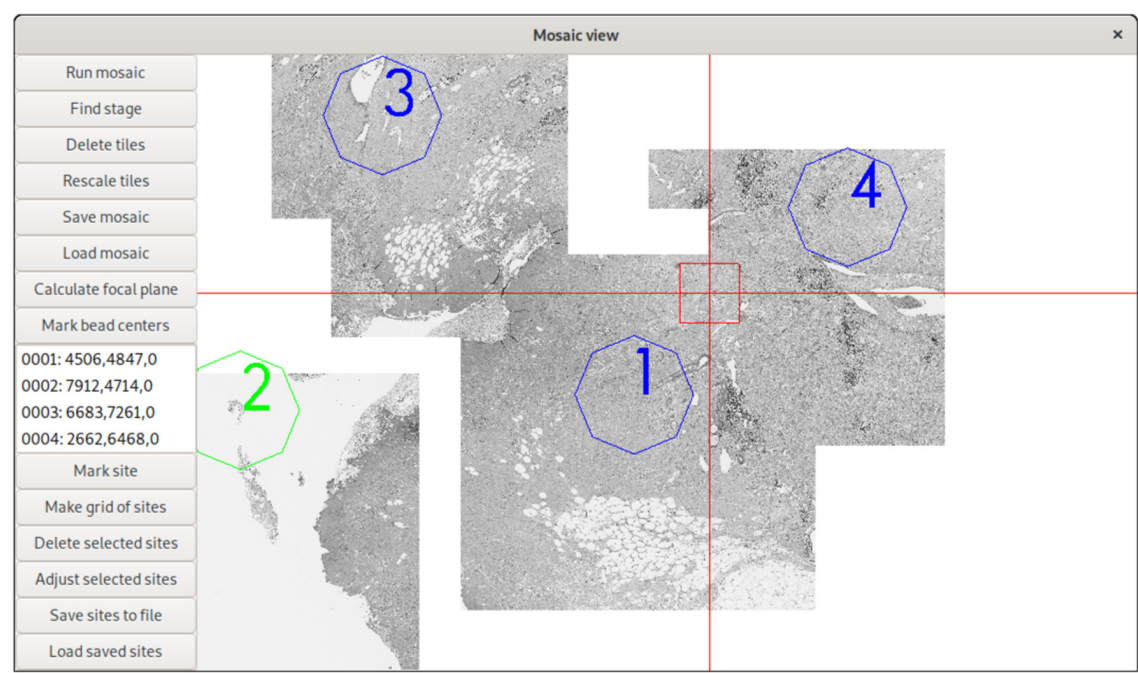

(d) Mosaic View

Figure 1. The main Cockpit Graphical User Interface (GUI) components: (a) The main window provides quick access to a number of functions and shows the status of all the devices, as well as the channels buttons to load predefined configurations. (b) The macro stage window provides an overview of the position of all stages, including nested stages and Z position. (c) The camera view shows the last image taken from each active camera and their histograms. (d) The mosaic view displays all mosaic images taken, saved locations, and dramatically eases navigation.

The simple interface design (Figure 1) enables users to focus on collecting the data they require without having to devise complex control infrastructure for systems of any level of complexity. It also enables easy navigation of even large sample regions. This can be especially important on bespoke microscopes which often lack eyepieces. For these types of system, Cockpit includes a touchscreen-optimised display window, providing large buttons and simple control over most functions (Figure 3).

The principal design goals for the Cockpit interface were to:

- Provide an easy-to-use, simple "Cockpit" control program for a wide range of microscopes.
- Hide from the user the complexity required to control an advanced microscope.

- Minimise the effort required to change hardware or integrate new hardware.

- Enable high-precision hardware timing signals to optimise experimental control, facilitate complex systems and maximise speed.

Cockpit provides an integrated microscope control package that enables all these features while being user friendly and portable across many different microscopes and common operating systems. 


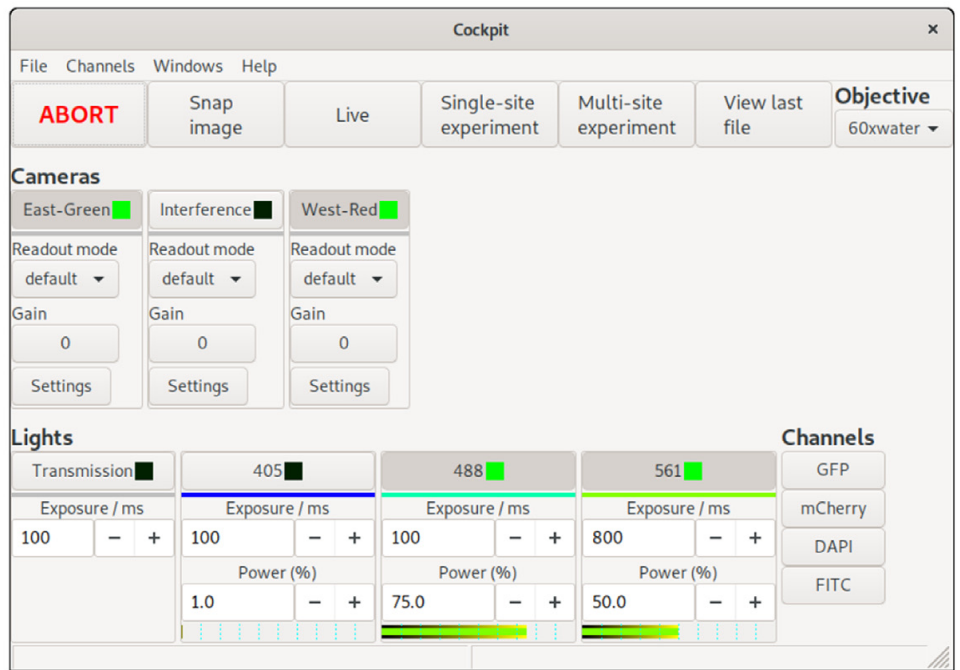

(a) GNU/Linux (Gnome)

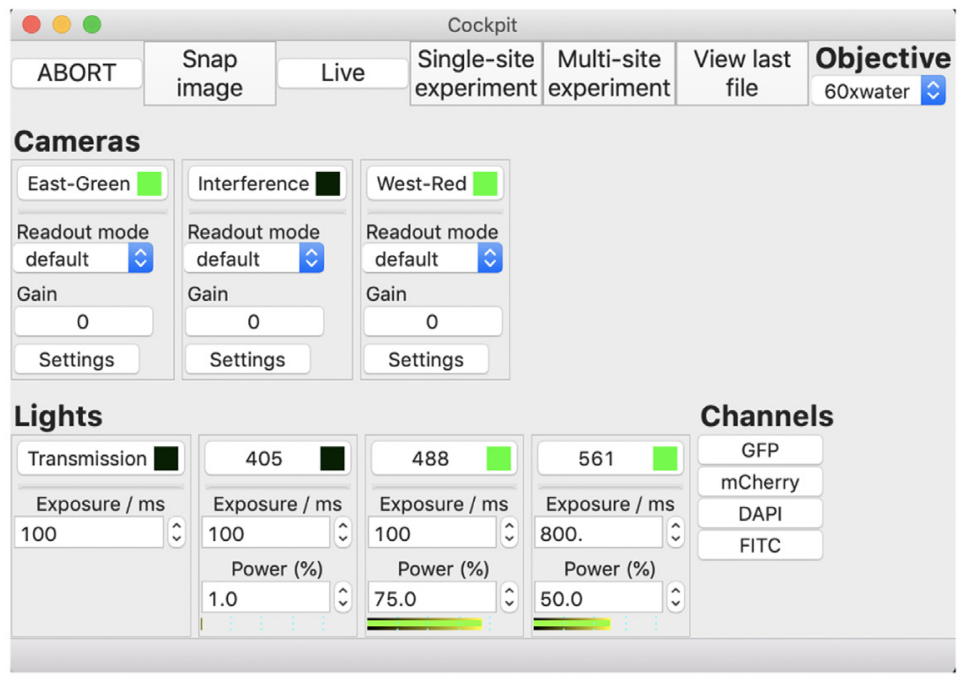

(b) $\operatorname{macOS}$

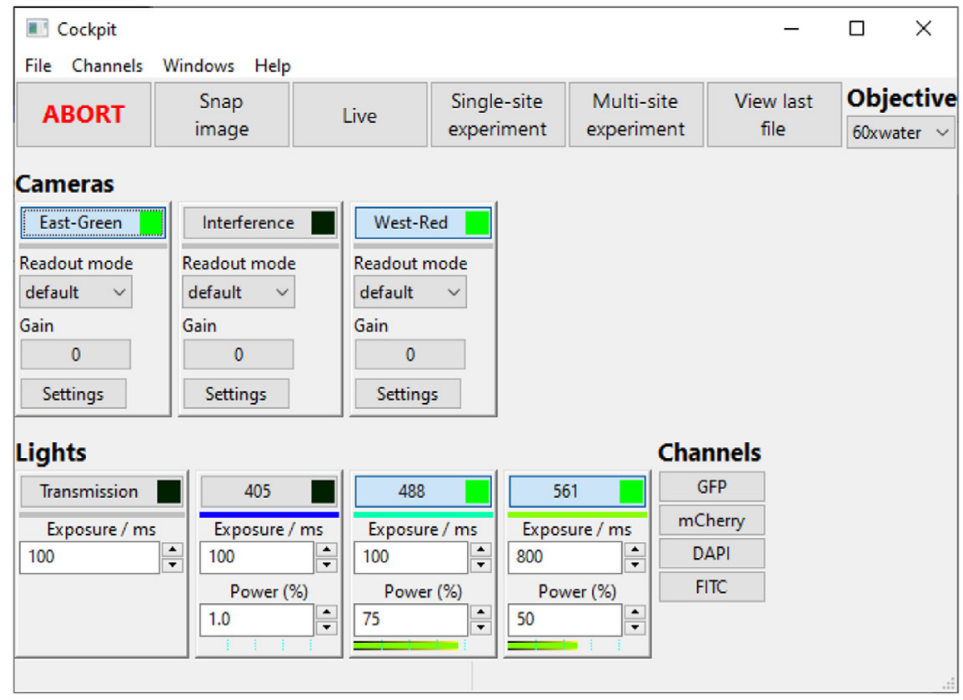

(c) Windows 10

Figure 2. The Cockpit main window under different operating systems. 


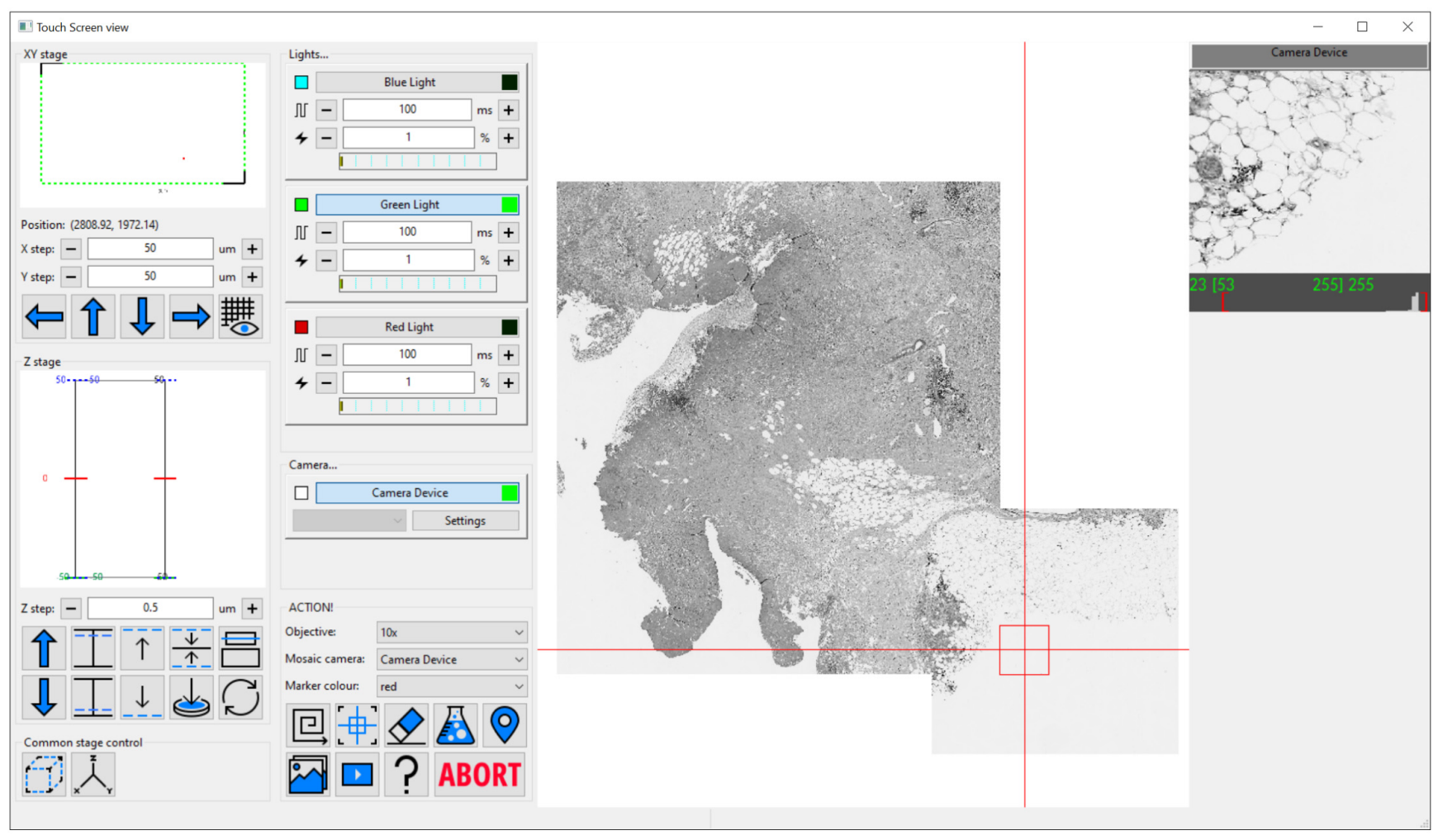

Figure 3. The Cockpit touchscreen window with the main GUI components in one window and large touchscreen friendly buttons. The left panel houses stage position indicators and controls, next panel over has devices like cameras and lights along with a set of large buttons at the bottom to run mosaics, centre the field of view on the current stage position, erase mosaic images, open the experiment dialog, drop a marker at the current position, display live video, run an experiment, a help button, and finally abort which stops an experiment or mosaic. The majority of the window is the mosaic display panel which can zoom from an overview up to the level of single pixels. Finally on the right is the last snapped image from each enabled camera, only one in this case.

The first version of the Cockpit software was developed in UCSF to drive the OMX microscope $e^{1,15,16}$, which was later commercialised. This code was re-written at UCSF and later released as open source under a BSD license. From this concept we have extensively developed the implementation of Cockpit to realise its full potential.

\section{Implementation}

Cockpit is implemented in the Python programming language ${ }^{24}$. The code consists of four main components: devices, handlers, interfaces, and the Graphical User Interface (GUI). Devices represent the individual physical devices, handlers represent control components of the devices, handlers from single or multiple devices are aggregated into interfaces, and the GUI component allows user interaction with the different interfaces.

\section{Devices and handlers}

The hardware control side of Cockpit has two parts: devices and handlers. A Cockpit device maps to a single physical device while handlers abstract the control components of the device to be used. For example, an XY stage device provides two handlers, one per axis. A laser device also provides two handlers, a light source handler and a light power handler: the light source handler controls the light source state - on or off — while the light power handler controls its intensity.

Most of the Cockpit code does not interact with Cockpit devices directly. Rather, when the Cockpit program starts, it constructs a "depot" object whose role is to obtain and keep track of the different device handlers. This architecture enables rapid changes in the choices of hardware devices, so they can be very easily added or removed from a complex system as it is being developed. This approach prevents potential conflicts or confusions such as addressing devices that do not exist or adding devices that lack drivers.

Within this architecture, any Python class can be used as a device; it is only required to provide one or more Cockpit handler. Built-in Cockpit devices include adaptors for the Python-Microscope package, thus supporting all of its available devices. Cockpit also supports the Aerotech PRO115 linear stage from the Soloist CP Controller, the PI M-687 XY stage from the PI C-867.262 controller, the Picomotor controller 8743-CL, the small 512x512 Spatial Light Modulator (SLM) and liquid crystal polarization rotator from Meadowlark 
Optics, and the SR470 laser shutter system from Stanford Research Systems.

Cockpit itself does not require a direct connection to the hardware. Indeed, most Cockpit devices are implemented as remote objects with the help of Pyro, a Python package for remote procedure calls. The use of Pyro enables the distribution of devices over the network to different computers, even running different operating systems.

\section{Interfaces and GUI}

Cockpit defines interfaces that are high-level abstractions for the system, with each controlling one or more handlers. For example, the stageMover interface is a unified translation manager that coordinates all translation stages, hiding the complexity of handling different stages for different axes and nested stages on the same axis. For instance, a system may include an $\mathrm{XY}$ stage, a coarse $\mathrm{Z}$ stage, and a high precision fast piezo $\mathrm{Z}$ stage. The GUI component provides different views onto the multiple interfaces and handlers, each exposing different levels of complexity.

Cockpit has a multi-window GUI, with each window serving a different purpose (Figure 1). This provides the flexibility to distribute the windows over multiple monitors in whatever form best fits the system being used. The main window provides an at-a-glance view of the state of the various devices; the mosaic window enables navigation of the sample relative to an overview formed of tiled images; the macro stage window displays the position of the different stages; and the camera view the current images from all active cameras. In addition, Cockpit also has a simpler single-window touchscreen interface (Figure 3) which duplicates a subset of the functionality provided on the other windows. This window is optimised for touchscreen interaction with large buttons for the most useful subset of functionality.

Cockpit uses wxPython, a Python wrapper to wxWidgets, to provide a user interface that has a native look and feel on the different platforms (Figure 2). Within wxPython, we make extensive use of OpenGL to enable high performance display and interaction with large images or image mosaics up to gigapixel sizes.

\section{Data acquisition experiments}

In Cockpit, the acquisition of an image series, such as a Z-stack or a time lapse sequence, is defined by an experiment. Cockpit experiments are based around the concept of an action table which must be run with hardware triggers. The experiment settings are converted into a list of device actions, which is parsed into a list of pre-computed analogue values and digital levels for each time point on a timing device. The action table is uploaded to the timing device and then started, running the experiment utilising hardware triggers and analogue voltages, where appropriate, to control all active devices. This approach, adapted from Carlton et al. ${ }^{1}$, is able to produce both digital triggers and analogue signals with precise timing.
Effective execution of the experiment requires accurate timing. In order to ensure that experiment timing is independent of the host computer's performance or the Python implementation, the timing functionality is performed using dedicated hardware. This enables time-critical operation of the microscope imaging tasks. The timing system has been implemented on several different hardware platforms, a digital signal processing board (Innovative Integration M67-160 with an A4D4 daughter board), an NI FPGA board (National Instruments cRIO-9068), and a Red Pitaya single board computer (STEMlab 125-14). In this way, we have created a universal and adaptable microscope platform with outstanding timing precision and accuracy.

System requirements

Cockpit requires Python 3.5 or later, and the Python packages PyOpenGL, Pyro4, Freetype Python, Matplotlib (RRID:SCR_ 008624) ${ }^{25}$, Python-Microscope, NumPy (RRID:SCR_008633) ${ }^{26}$, pySerial, SciPy (RRID:SCR_008058) ${ }^{27}$, and wxPython. These are all free and open-source software that are available for all widely-used operating systems. Hence, Cockpit can be used across GNU/Linux, macOS, and Windows.

The Cockpit interface incorporates some assumptions as to what is required for a minimal microscope. It requires at least one camera, a computer controlled $\mathrm{X}, \mathrm{Y}$ and $\mathrm{Z}$ translation motorised axes, and a light source that can either be hardware triggered (directly or via a shutter) or left on during an experiment. In addition, experiments require a device that can be programmed as source of hardware triggers, supplying digital signals and, optionally, analogue voltages.

With no configuration to specific hardware, Cockpit will automatically start in simulation mode, providing simulated cameras, light sources, and stages. This allows testing and demonstration of the software without any hardware or configuration. This can be further extended by utilising the test devices within the associated Python-Microscope package. In this mode, with some configuration, the software can link simulated $\mathrm{XYZ}$ stage, camera, and filter-wheel to return a channel defined by the filter-wheel and a subregion defined by the stage position from a large multi-channel image. The simulation is further improved by blurring the image based upon the $\mathrm{Z}$ position of the stage. This mode was used for Figure 1 and Figure 3 in this manuscript.

\section{Use cases}

Cockpit is optimised to provide an effective user experience running a fully automated microscope on systems with no eyepieces. A significant component of this is powerful mapping and overview tools that provide a guide-map to the specimen landscape, which is interactive and highly responsive. The system can collect arbitrary mosaic images, which are acquired in an expanding spiral pattern. Acquired mosaic images are immediately uploaded to the graphics card as a texture map. This allows smooth and rapid display of these images from low zoom overview all the way to single pixel views. The graphics card can then render these images interactively in real time. 
The typical texture memory on modern graphics cards mean that the system can navigate in real time around mosaics made from hundreds or thousands of individual images, with resulting mosaics up to gigapixels in size. Starting a new mosaic does not remove previous images, so multiple, possibly overlapping, mosaic areas can be collected. Keeping previous mosaic images means it is easy to navigate multiple regions of the sample without having to reacquire overview images. This greatly eases the navigation of complex samples.

There are two obvious use cases for this functionality. In the first case, one could utilise a low magnification lens to map a large section, or even the whole of the sample area, say a coverslip as shown in Figure 4(a). This produces a $\sim 250$ megapixel image, via which the user can visit any site of choice and re-image points in fluorescence mode with higher magnification objectives (Figure 4(b)). The new detailed images are then "painted" onto the guide-map in real-time. As the second case, in samples such as tissue culture cells, where many similar regions are available, smaller regions can be imaged at higher resolution to select interesting targets for further imaging. The preservation of previous mosaics when a new one is initiated is a critical component of this process, as it allows easy navigation of multiple sample regions. It is then practical to return to the regions of most interest without requiring reimaging, which would waste time and incur unnecessary light exposure to the sample.

To improve the user-centred microscope experience, we divided the use of the microscope into two stages. The user starts an imaging session by rapidly exploring large regions or even the entire slide to create a guide map, while marking regions of interest for later inspection. In the first stage, the touchscreen can be used to build the guide map. The user then establishes the conditions required for the desired images, such as choice of laser lines and their intensities, exposures, number of channels and cameras, $\mathrm{Z}$ sectioning range, and points to visit on the guide map. The use of a touchscreen enables rapid setting up of the conditions for the experiment, focusing on the most important features of the microscope hardware without providing full functionality.

In a second phase of acquisition, a more detailed software control window is used with a conventional keyboard to define any more advanced experimental settings, such as numeric values for time lapse interval which are difficult to set from a touch interface. Finally the experiment is run.

In this way, we have created a unique, near-instantaneous control environment that allows the user to start an imaging session by exploring the entire slide quickly and marking regions to visit and image in order to achieve their scientific goals. Our software provides a novel way for the user to plan their entire work flow rapidly and with efficient specimen usage.

\section{Previously published systems and microscopes in development using Cockpit}

To exemplify the flexibility of Cockpit, we present a number of systems that currently run using the software as the user interface. Along with previously published systems, we have a number of microscopes at earlier stages of development where Cockpit provides the user interface.

Cockpit has already been deployed in three systems reported in previous publications. The first is a cryo-super resolution microscope for correlative imaging, CryoSIM ${ }^{28,29}$ (Figure 5(a)).

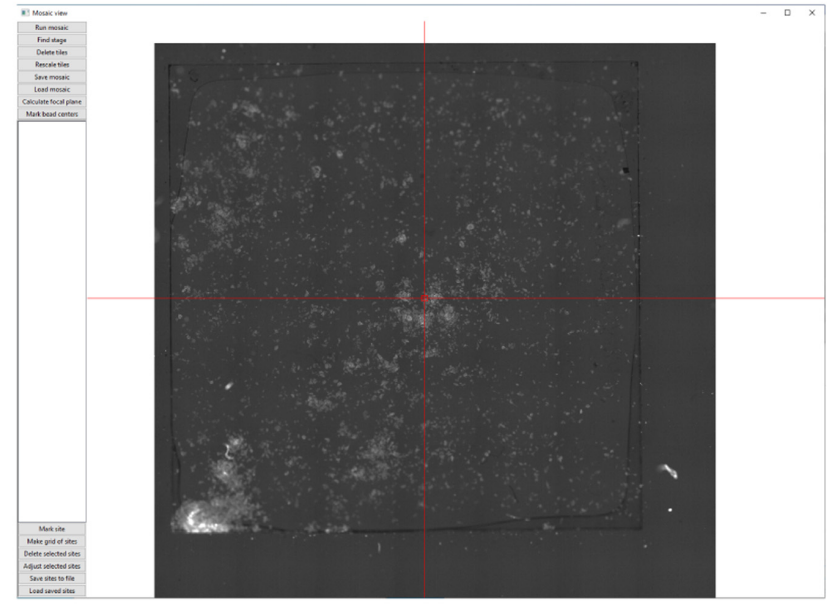

(a) Complete coverslip imaged with a $4 \times$ objective

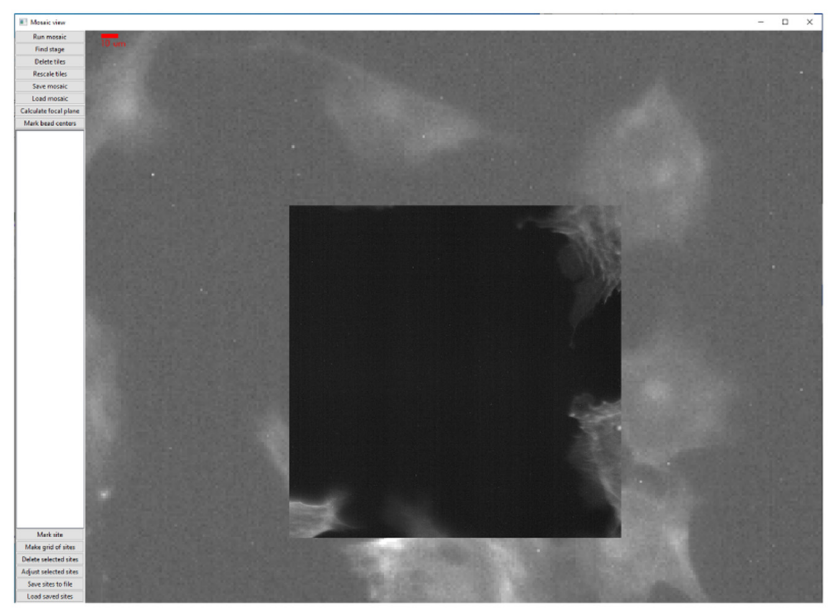

(b) Magnified portion with region images with a $63 \times$ objective

Figure 4. The mosaic tool used to image a standard Thermo Fisher test slide (FluoCells ${ }^{\mathrm{TM}}$ Prepared Slide \#1, F36924) with: (a) mosaic of a complete coverslip imaged with a $4 \times$ objective (pixel size $1.502 \mu \mathrm{m}$ ) consisting of more than 250 megapixels; (b) a magnified portion of the mosaic with a single image from a $63 \times$ objective (pixel size $0.0954 \mu \mathrm{m}$ ) painted in on the low resolution map. The higher magnification image has much better signal to noise ratio from better light collection as the objective has a much higher numerical aperture. 


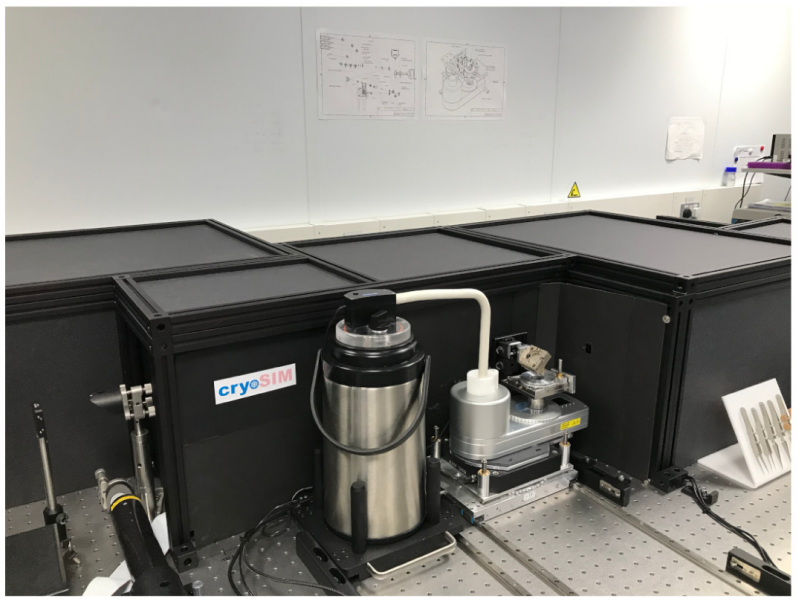

(a) CryoSIM

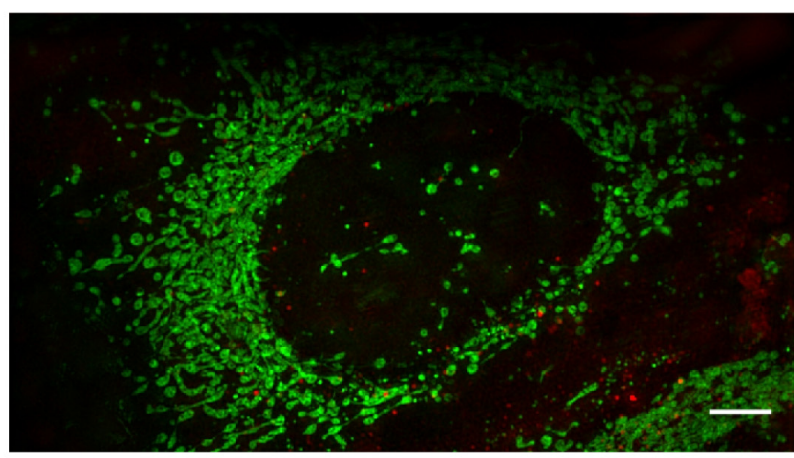

(c) Image from CryoSIM

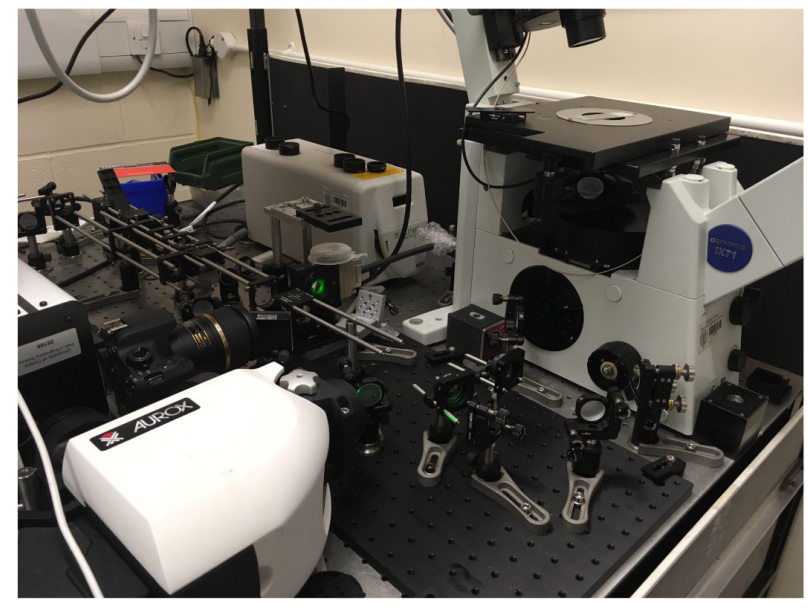

(b) Aurox AO

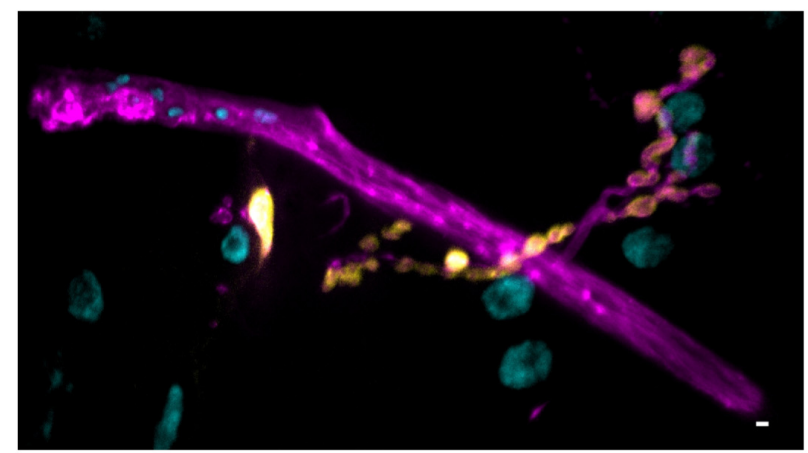

(d) Image from Aurox $\mathrm{AO}$

Figure 5. Previously published systems that use Cockpit: (a) the CryoSIM system; (b) the Aurox Clarity AO laser free spinning disk confocal system; (c) image acquired on CryoSIM of cryo preserved HeLa cell labelled with MitoTracker green and LysoTracker red; (d) image acquired on the Aurox Clarity system with AO correction at high optical sectioning. Image is a maximum intensity projection of a $20 \mu \mathrm{m} z$-stack. Sample was a Drosophila neuromuscular junction with DLG in yellow, HRP in magenta, and DAPI in cyan. Scale bars $5 \mu \mathrm{m}$.

The second demonstration was a laser-free spinning disk confocal microscope using adaptive optics (AO) for aberration correction $^{30}$ (Figure 5(b)). Additionally, Cockpit was the basis of the control software in the implementation of a novel technique, IsoSense, for improved aberration correction in widefield microscopes and structured illumination ${ }^{31}$.

\section{A simple widefield microscope}

We present a simple, compact, fully automated, portable inverted microscope based around the Zaber MVR system. This provides a bare-bones inverted stand with motorised $\mathrm{X}, \mathrm{Y}$, and $\mathrm{Z}$ axes, a multi-position motorised filter turret, and LED-based fluorescence illumination. The system has a Ximea camera with a small physical footprint (approximately $30 \mathrm{~mm}$ cube) and a Red Pitaya single board computer running as a hardware timing device (Figure 6).

This system is able to take fluorescence images in three colours, using the three illumination LEDs and a quad-bandpass dichroic filter set. The system has $4 \times$ and $63 \times$ air objectives.
Although changing the objective involves physically removing the objective and its mount, this is easily achieved due to the spring-loaded kinematic mounting system. The Cockpit mosaic function allows large areas of a sample to be mapped quickly (Figure 4(a)), and then regions of interest can be selected and marked for return later or the mosaic stopped and z-stacks in one or more channels collected. If a live sample is used the system can be used for time lapse imaging.

This system provides a demonstration of the power and portability of Cockpit and it functions as a test bed for new software developments on practical hardware. The system's small size and portability enable easy transport of the system for demonstrations and collaborative projects in other laboratories.

\section{A complex structured illumination microscope with adaptive optics}

In a further system, we are using Cockpit to maximise the performance of complex upright widefield structured illumination 


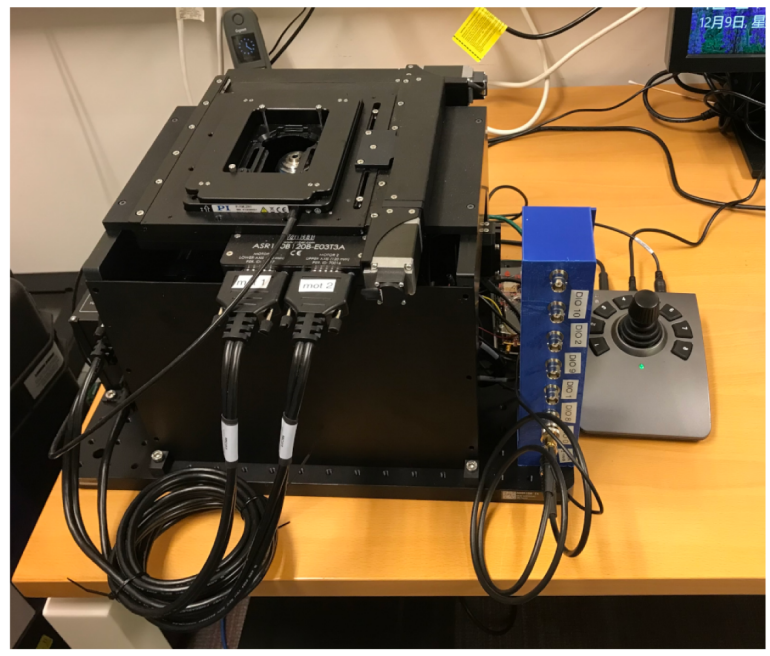

(a) Zaber system

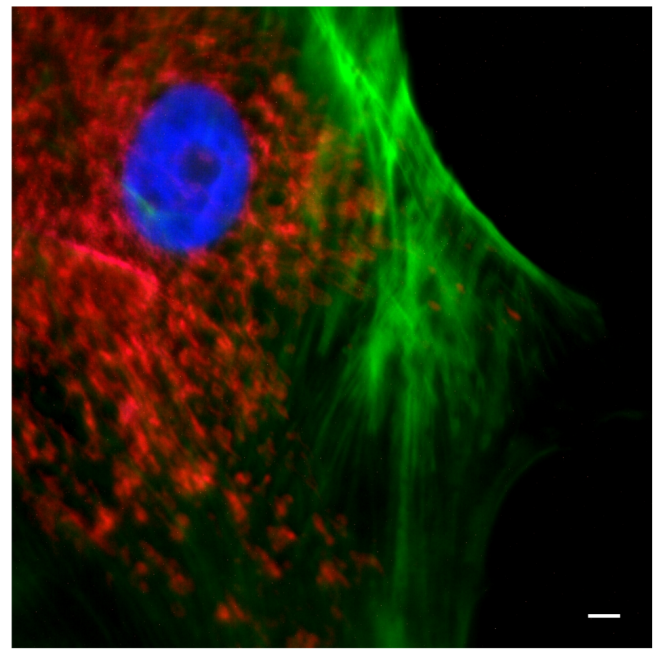

(b) 3 colour image

Figure 6. The Zaber microscope. (a) An image of the system, showing its compact size with a 300×450 mm footprint, (b) a three colour image showing the nucleus stained with DAPI in blue, the actin in green and mitochondria stained with MitoTracker Red CMXRos in Red. Scale bar $5 \mu \mathrm{m}$.

microscope, referred to as DeepSIM (Figure 7(a)). This incorporates not only translation stages, light sources, and cameras, but also a spatial light modulator (SLM) and a deformable mirror (DM) for AO. This set-up is required to perform super-resolution live imaging experiments deep in tissue specimens, such as on the Drosophila larval neuro-muscular junction preparation, a powerful model system for understanding synapse biology on a molecular level. In order to achieve this imaging the system has to synchronise the illumination lasers, the SLM, a polarisation rotator, the DM, the $\mathrm{Z}$ stage position, and the cameras. The lasers, SLM, DM, and cameras receive digital triggers from the Red Pitaya, while the Z-position and polarisation rotator state are controlled via analogue voltages, all synchronised at the $\mu$ s level. Cockpit provides a simple user interface allowing selection of different experimental parameters, such as exposure time, laser power, $\mathrm{Z}$ step, and stack size (Figure 7(b)). The experiment module creates the relevant signals and timing information, transfers this to the Red Pitaya which then controls the hardware during the experiment.

Cockpit also provides a user-friendly interface to Microscope-AOTools ${ }^{32}$, facilitating use of a wavefront sensor for calibration of the DM then adaptively correcting sample induced aberrations via image-based metrics, using so-called sensorless AO. This interface also implements IsoSense $^{31}$ to improve aberration detection over a wide range of complex samples.

Although this system is extremely complex with a multitude of devices the user interface is clean and easy to use. The system has sensible defaults, thus minimising the expertise and interaction required to collect experimental data.

\section{Discussion}

We have developed a Python-based GUI for controlling bespoke microscopes which can map the entire slide or dish, allowing a real time exploration of the entire specimen landscape. It also connects to separate hardware timing devices to enable high precision timing of even complex devices and experiments. A key property of our software is that it is focused around the needs of the user and their experimental design and workflow. Finally, using Python means that the microscope control can easily be modified to make use of the extensive machine learning algorithms available in easy to integrate libraries. This means that a microscope system that is controlled by Cockpit can readily be adapted to make decisions using machine learning algorithms during the acquisition process in order to modify the imaging conditions.

\section{Existing control options}

Cockpit has been introduced as an alternative to a number of existing microscope control options that are already available. Most published systems take one of three approaches: 1) utilise individual software packages provided by the device manufactures, 2) use LabVIEW to integrate LabVIEW-based drivers (VIs) provided by the manufacturers into a single custom GUI, or 3) use the open source microscope control package $\mu$ Manager $^{33}$. We will discuss the relative merits of each of these options in turn.

Using individual software packages to control each device is simple and direct. However this approach has several severe drawbacks. Each hardware device needs its own software, which takes up screen space and computer resources. This also 


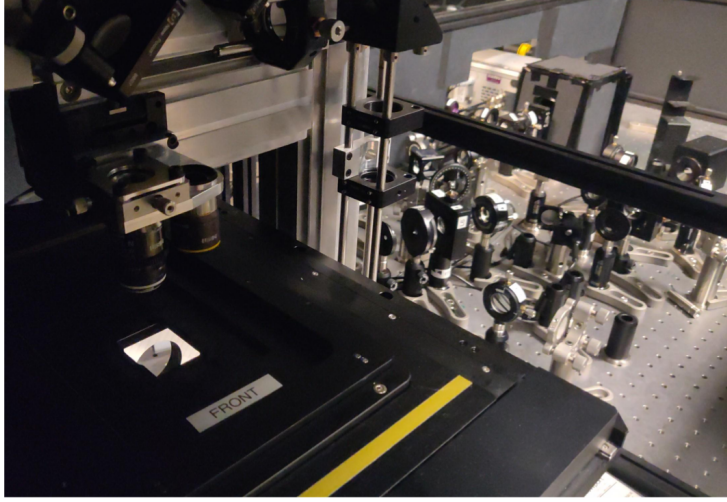

(a) DeepSIM

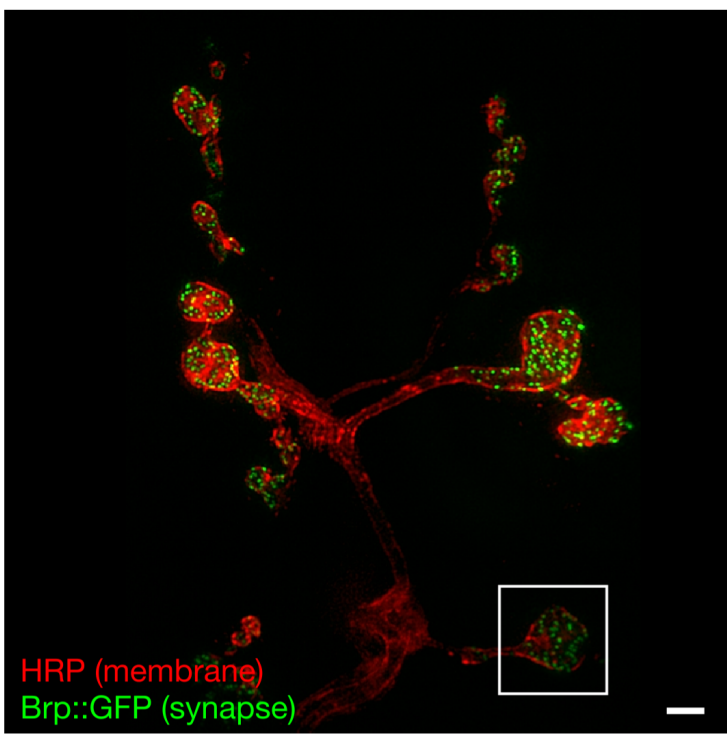

(c) Full image

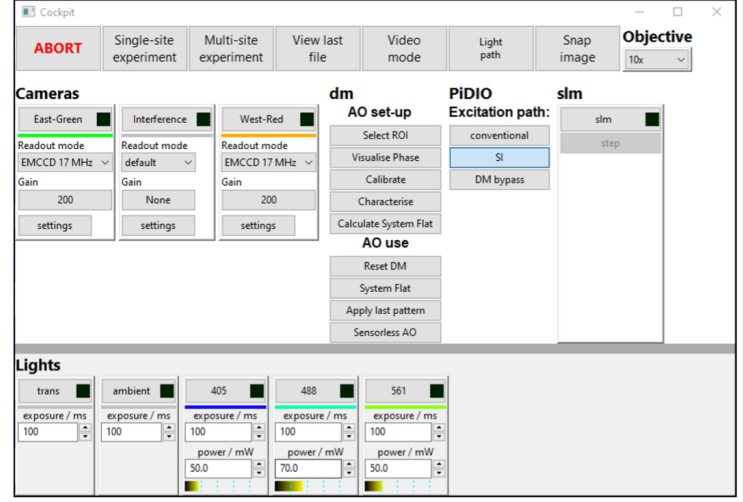

(b) Cockpit main window with $\mathrm{AO}$ tools

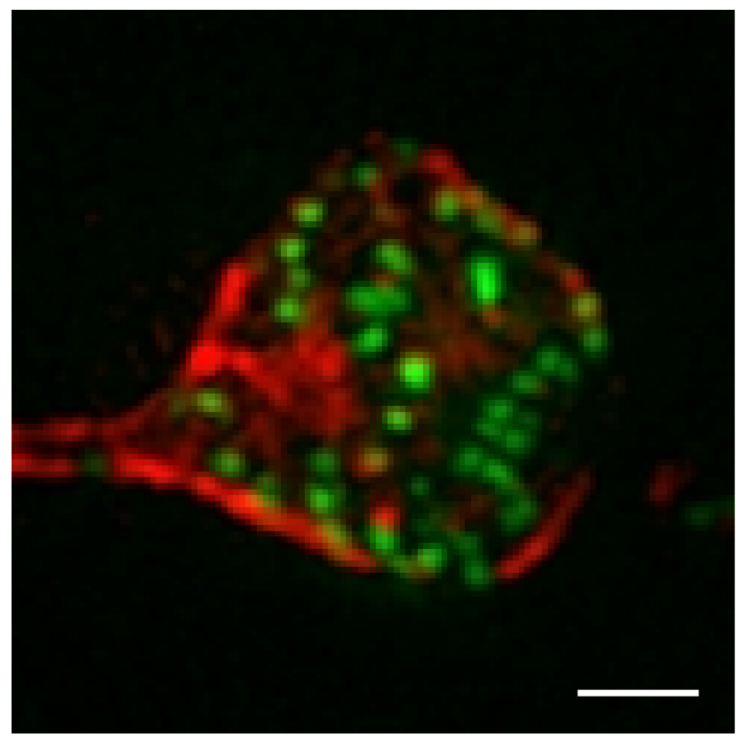

(d) Inset of (c)

Figure 7. Example data from the DeepSIM system using Cockpit to image deep ( $20 \mu \mathrm{m})$ in Drosophila neuro-muscular junctions. The system uses sensorless AO to correct for sample induced aberrations. (a) system view from the stage; (b) main Cockpit window with the additional controls for the SLM and AO devices; (c) image of individual synapses at the Drosophila neuro-muscular junction using AOSIM imaging, with Cy3 labelled HRP antibody labelling the neuronal membrane in red and Brp::GFP in the synapse in green; (d) zoomed in region of $(\mathbf{c})$. Scale bars are $2 \mu \mathrm{m}$.

removes the ability of different hardware parts to automatically interact with each other, for instance changing a stage's position once a camera has finished collecting images. Producing Z-stacks, or multi-channel images on such a setup is awkward, slow and open to human errors. These factors can lead to corruption of the results.

LabVIEW is a visual programming tool that allows the construction of "programs" by connecting modules with wires and building graphical interfaces. Many manufacturers provide software to allow their hardware to be used in LabVIEW, through so-called virtual instruments (VIs). Building clean and simple visual framework to control highly complex advanced optical systems in LabVIEW requires advanced expertise in the program's use. Because such skills are usually beyond the ability of most scientists in an academic setting, many one-off complex bespoke systems, built to demonstrate a new principle, are very hard to use routinely by experimental scientists. Despite their obvious importance, such systems can rarely be reproduced and adopted by others for routine use.

$\mu$ Manager is an open source generalised microscope control interface which works on top of ImageJ, a popular image analysis program. ImageJ is written in Java, and $\mu$ Manager is written in a combination of Java and $\mathrm{C}++$. $\mu$ Manager's approach is most directly comparable to Cockpit. Many hardware device manufacturers provide $\mu$ Manager compatible libraries and instructions on how to connect and control their hardware. The 
package comes with mechanisms to run basic experiments, and the ability sequence commands.

Being based on Image J, $\mu$ Manager, by design, includes a large application with multiple windows. This also means that the majority of the interface must be written in Java; however the system also needs a $\mathrm{C} / \mathrm{C}++$ layer to interface the Java code to $\mathrm{C} / \mathrm{C}++$ based system libraries. Cockpit is written in pure Python, relying on the Python-Microscope package for hardware interfacing. Producing a strict separation of the user interface from the hardware control components. This has the additional benefit that connected hardware may be physically located on another computer, increasing scalability and allowing devices requiring incompatible hardware or software to still be seamlessly integrated.

The mosaic window in Cockpit is similar in concept to Micro-Magellan $^{23}$, a $\mu$ Manager plugin. Being able to record a large area view, possibly from multiple areas, and have that available for instant navigation. The mosaic functionality dramatically speeds up experiment setup and finding the correct regions of interest, especially on bespoke systems with no eyepieces. Our mosaic interface utilises modern fast GPUs to enable instant access to even very large mosaic maps. Additionally, the touch screen interface allows easy and intuitive access to most of the functionality using a visual grammar that is very familiar to everyone.

The dedicated timing device interface allows fast and repeatable timing for both simple and complex experiments. It is useful in general but absolutely indispensable for experiments like the live cell adaptive optics SIM experiment (Figure 7(c)).

The standardised device interfaces from Python-Microscope mean that replacing one device with another of the same type is simply a matter of changing the address of the device in a configuration file.

Cockpit is still under active development, both within our labs and at several other laboratories across multiple countries. We are working on adding online image analysis to further enhance the mosaic functionality with machine learning, enabling the system to capture a large area of the sample quickly at low-resolution and then automatically identify features of interest for 3D-multi-channel acquisition.

\section{Conclusions}

We have developed Cockpit, a new paradigm for user-based software control of complex bespoke microscopes. The software is highly adaptable by a user or engineer with experience in Python. The key advantage of our approach is that a user is presented with a clean and simple interface that hides the complexity of the hardware, so they can focus on their experimental design and obtain data from the instrument with a high precision and reproducible workflow. We hope that the community will adopt this package and help us to continue to develop it.

\section{Methods \\ CryoSIM imaging}

The CryoSIM system has previously been published in detail $^{28,29}$. It was used to image HeLa cells grown on carbon coated gold EM grids under standard tissue culture conditions. Cells were labelled with MitoTracker Green (Thermo Fisher) at $100 \mathrm{nM}$ and LysoTracker Red DND-99 (Thermo Fisher) at $50 \mathrm{nM}$ after 30 minutes of incubation, to give green mitochondria and red lysosomes. The grids with live cells on them were then blotted and plunge frozen in liquid nitrogen cooled liquid ethane (Leica EM GP2) before being transferred to liquid nitrogen storage. Once frozen, grids were preserved in liquid nitrogen or at cryogenic temperatures on the imaging systems to prevent thawing and detrimental ice crystal formation. Structured Illumination Microscopy (SIM) images were collected with $488 \mathrm{~nm}$ and $561 \mathrm{~nm}$ laser excitation and emission collected at $525 / 50$ and $605 / 70$ on two Andor iXon EMCCD cameras. Images were reconstructed using SoftWoRx (GE Healthcare) and image quality was assessed with SIMcheck ${ }^{34}$.

\section{Aurox AO imaging}

The Aurox Clarity AO system is as previously published, the system utilised an Olympus IX70 microscope with a $60 \times 1.42 \mathrm{NA}$ objective. Illumination was provided by a CoolLED p-300 30 . This system was used to image Drosophila melanogaster neuro-muscular junctions (NMJ). The samples were prepared by following the protocol form Brent et al. $2009^{35}$. 3rd instar Drosophila melanogaster larvae (Oregon-R strain) were dissected in HL3 buffer with $0.3 m M \mathrm{Ca}^{2+}$ to prepare a larval fillet. After this, larvae were fixed with $4 \%$ paraformaldehyde and blocked using $1 \% \mathrm{BSA}^{35}$. Larvae were stained overnight with 1:100 Horseradish Peroxidase (HRP) conjugated to Alexa568 fluorophore to visualise the neurons, and primary mouse antibody against Discs large (DLG) to visualise the postsynaptic density. The next day, the larvae were counter stained with secondary antibody to detect the DLG (1:200 donkey anti-mouse conjugated to Alexa488 fluorophore was used), as well as 1:1000 from $1 \mathrm{mg} \mathrm{mL}^{-1}$ stock DAPI to visualise the nuclei. The larvae were then washed and mounted in $65 \%$ vectashield.

\section{Zaber imaging}

This system is based around a Zaber MVR motorised inverted microscope with Zeiss optics and 4x, 0.13NA and 63x 0.75 Air objectives. Fluorescence illumination comes via 3 LEDs (385 nm, $473 \mathrm{~nm}$ and $568 \mathrm{~nm}$ ) and a quad bandpass dichroic filter set (Chroma \#89402). The system has a small format (approx $30 \mathrm{~mm}$ cube) Ximea (model MQ042MGCM) camera and a Red Pitaya single board computer running as a hardware timing device. Multi channel images were taken by sequentially illuminating with the three different LEDs without changing the quad dichroic filter cube.

\section{DeepSIM imaging}

DeepSIM is a custom made upright structured illumination microscope with AO specifically designed for SIM super resolution imaging deep into live tissue (manuscript in preparation). 
On the DeepSIM system images were taken using $488 \mathrm{~nm}$ and $561 \mathrm{~nm}$ laser illumination. The system includes a Structure Light Modulator (SLM, Meadowlark) to provide structured illumination for SIM imaging. The system also includes a deformable mirror (Alpao DM-69) for aberration correction. The system has a $60 \mathrm{x} 1.1$ water dipping objective and samples were imaged in aqueous buffer. The AO components were calibrated and controlled using Microscope-AOTools ${ }^{32}$ to reduce aberrations and produce good SIM imaging at depth in biological samples.

The samples were prepared by following the protocol ${ }^{35}$. 3rd instar Drosophila melanogaster larvae (Bruchpilot (Brp)-GFP strain) were dissected in HL3 buffer with $0.3 m M \mathrm{Ca}^{2+}$ to prepare a so-called larval fillet, and the larval brains were removed. After this, larvae were stained for 15 minutes with 1:50 Horseradish Peroxidase (HRP) conjugated to $\mathrm{Cy} 3$ fluorophore to visualise the neurons, washed with HL3 buffer with $0.3 m M \mathrm{Ca}^{2+}$ and imaged in HL3 buffer with $0 m M \mathrm{Ca}^{2+}$ to prevent the larvae from moving.

\section{Data availability}

No data are associated with this article.

\section{Software availability}

Software available from: https://pypi.org/project/microscopecockpit/
Source code availabile from: https://github.com/MicronOxford/ cockpit

Archived source code at time of publication (version 2.9.1): https://zenodo.org/record/454286324

Cockpit is distributed under the terms of the GNU General Public License as published by the Free Software Foundation, either version 3 of the License, or (at your option) any later version.

\section{Acknowledgements}

We are grateful to the original UCSF software team led by John Sedat, including Melvin Jones, Chris Weisiger and Sebastian Haase for the initial writing of the Python code used to control the OMX and OMX-T microscopes, which was subsequently released as open source software and formed the starting point for this project. We wish to thank many colleagues who have provided helpful suggestions, discussions and testing during development of Cockpit, the Micron Advanced Bioimaging Unit particularly Mantas Žurauskas and Andrew Jefferson, Maria Harkiolaki from the Diamond Light Source beamline B24. Oscar Davis for help on the interface look and feel. An earlier version of this article can be found on bioRxiv (https://doi.org/10.1101/2021.01.18.427171).

\section{References}

1. Carlton PM, Boulanger J, Charles K, et al.: Fast live simultaneous multiwavelength four-dimensional optical microscopy. Proc Natl Acad Sci US A. 2010; 107(37): 16016-16022.

PubMed Abstract | Publisher Full Text | Free Full Text

2. Stelzer EHK: Light-sheet fluorescence microscopy for quantitative biology. Nat Methods. 2015; 12(1): 23-26. PubMed Abstract | Publisher Full Text

3. Chen $B C$, Legant WR, Wang $K$, et al.: Lattice light-sheet microscopy: imaging molecules to embryos at high spatiotemporal resolution. Science. 2014; 346(6208): 1257998

PubMed Abstract | Publisher Full Text | Free Full Text

4. Gustafsson MG: Surpassing the lateral resolution limit by a factor of two using structured illumination microscopy.J Microsc. 2000; 198(Pt 2): 82-87. PubMed Abstract | Publisher Full Text

5. Pitrone PG, Schindelin J, Stuyvenberg L, et al.: OpenSPIM: an open-access light-sheet microscopy platform. Nat Methods. 2013; 10(7): 598-599. PubMed Abstract | Publisher Full Text | Free Full Text

6. Markwirth A, Lachetta M, Mönkemöller V, et al: Video-rate multi-color structured illumination microscopy with simultaneous real-time reconstruction. Nat Commun. 2019; 10(1): 4315. PubMed Abstract | Publisher Full Text | Free Full Text

7. Zdankowski P, McGloin D, Swedlow JR: Full volume super-resolution imaging of thick mitotic spindle using 3D AO STED microscope. Biomed Opt Express. 2019; 10(4): 1999-2009.

PubMed Abstract | Publisher Full Text | Free Full Text

8. Eilers $\mathrm{Y}, \mathrm{Ta} \mathrm{H}, \mathrm{G}$ wosch $\mathrm{KC}$, et al.: MINFLUX monitors rapid molecular jumps with superior spatiotemporal resolution. Proc Natl Acad Sci U S A. 2018. 115(24): 6117-6122.

PubMed Abstract | Publisher Full Text | Free Full Text

9. Holden SJ, Uphoff S, Kapanidis AN: DAOSTORM: an algorithm for highdensity super-resolution microscopy. Nat Methods. 2011; 8(4): 279-280. PubMed Abstract | Publisher Full Text
10. Marsh RJ, Pfisterer $K$, Bennett $P$, et al.: Artifact-free high-density localization microscopy analysis. Nat Methods. 2018; 15(9): 689-692. PubMed Abstract | Publisher Full Text

11. Rosten $\mathrm{E}$, Jones $\mathrm{GE}$, Cox $\mathrm{S}$ : ImageJ plug-in for bayesian analysis of blinking and bleaching. Nat Methods. 2013; 10(2): 97-98. PubMed Abstract | Publisher Full Text

12. Cnossen J, Hinsdale $T$, Thorsen R precision with patterned illumination. Nat Methods. 2020; 17(1): 59-63. PubMed Abstract | Publisher Full Text | Free Full Text

13. Nadella KMNS, Roš $\mathrm{H}$, Baragli $\mathrm{C}$, et al:: Random-access scanning microscopy for 3D imaging in awake behaving animals. Nat Methods. 2016; 13(12): 1001-1004.

PubMed Abstract | Publisher Full Text | Free Full Text

14. York AG, Chandris $P$, Nogare DD, et al.: Instant super-resolution imaging in live cells and embryos via analog image processing. Nat Methods. 2013; 10(11): 1122-1126.

PubMed Abstract | Publisher Full Text | Free Full Text

15. Dobbie IM, King E, Parton RM, et al.: Omx: A new platform for multimodal, multichannel wide-field imaging. Cold Spring Harb Protoc. 2011; 2011(8): 899-909.

PubMed Abstract | Publisher Full Text | Free Full Text

16. Schermelleh L, Carlton PM, Haase S, et al:: Subdiffraction multicolor imaging of the nuclear periphery with 3D structured illumination microscopy. Science. 2008: 320(5881): 1332-1336.

PubMed Abstract | Publisher Full Text | Free Full Text

17. Booth MJ: Adaptive optical microscopy: the ongoing quest for a perfect image. Light Sci Appl. 2014; 3(4): e165. Publisher Full Text

18. Liu TL, Upadhyayula S, Milkie DE, et al.: Observing the cell in its native state: Imaging subcellular dynamics in multicellular organisms. Science. 2018; 360(6386): eaaq1392.

PubMed Abstract | Publisher Full Text | Free Full Text 
19. Turcotte R, Liang $Y$, Tanimoto $M$, et al.: Dynamic super-resolution structured illumination imaging in the living brain. Proc Natl Acad Sci U S A. 2019; 116(19): 9586-9591.

PubMed Abstract | Publisher Full Text | Free Full Text

20. Gould TJ, Burke D, Bewersdorf J, et al.: Adaptive optics enables 3D STED microscopy in aberrating specimens. Opt Express. 2012; 20(19): 2099821009.

PubMed Abstract | Publisher Full Text | Free Full Text

21. Kner $P$, Winoto $L$, Agard DA, et al.: Closed loop adaptive optics for microscopy without a wavefront sensor. In: Three-Dimensional and Multidimensional Microscopy: Image Acquisition and Processing XVII. Proc SPIE Int Soc Opt Eng. 2010; 7570: 757006.

PubMed Abstract | Publisher Full Text | Free Full Text

22. Kner P, Sedat JW, Agard DA, et al.: High-resolution wide-field microscopy with adaptive optics for spherical aberration correction and motionless focusing. J Microsc. 2010; 237(2): 136-147.

PubMed Abstract | Publisher Full Text | Free Full Text

23. Pinkard H, Stuurman N, Corbin K, et al.: Micro-Magellan: open-source, sample-adaptive, acquisition software for optical microscopy. Nat Methods. 2016; 13(10): 807-809.

PubMed Abstract | Publisher Full Text | Free Full Text

24. Mick, Pinto DMS, Dobbie I, et al.: MicronOxford/cockpit release-2.9.1. (Version release-2.9.1) Zenodo. 2021

http://www.doi.org/10.5281/zenodo.4542863

25. Hunter JD: Matplotlib: A 2D graphics environment. Comput Sci Eng. 2007; 9(3): 90-95.

Publisher Full Text

26. Harris $\mathrm{CR}$, Millman $\mathrm{KJ}$, van der Walt SJ, et al:: Array programming with NumPy. Nature. 2020; 585(7825): 357-362

PubMed Abstract | Publisher Full Text | Free Full Text
27. Virtanen P, Gommers R, Oliphant TE, et al.: SciPy 1.0: Fundamental algorithms for scientific computing in python. Nat Methods. 2020; 17(3): 261-272. PubMed Abstract | Publisher Full Text | Free Full Text

28. Kounatidis I, Stanifer ML, Phillips MA, et al.: 3D correlative cryo-structured illumination fluorescence and soft $\mathrm{X}$-ray microscopy elucidates reovirus intracellular release pathway. Cell. 2020; 182(2): 515-530.e17.

PubMed Abstract | Publisher Full Text | Free Full Text

29. Phillips MA, Harkiolaki M, Pinto DMS, et al.: CryoSIM: super resolution 3D structured illumination cryogenic fluorescence microscopy for correlated ultra-structural imaging. bioRxiv. 2020 Publisher Full Text

30. Hussain SA, Kubo T, Hall N, et al.: Wavefront-sensorless adaptive optics with a laser-free spinning disk confocal microscope.J Microsc. 2020. PubMed Abstract | Publisher Full Text

31. Žurauskas M, Dobbie IM, Parton RM, et al.: IsoSense: frequency enhanced sensorless adaptive optics through structured illumination. Optica. 2019; 6(3): 370-379.

PubMed Abstract | Publisher Full Text | Free Full Text

32. Hall N, Titlow J, Booth MJ, et al:: Microscope-AOtools: a generalised adaptive optics implementation. Opt Express. 2020; 28(20): 28987-29003. PubMed Abstract | Publisher Full Text

33. Edelstein A, Amodaj N, Hoover K, et al.: Computer control of microscopes using umanager. Curr Protoc Mol Biol. 2010; 92(1): 14-20. PubMed Abstract | Publisher Full Text | Free Full Text

34. Ball G, Demmerle J, Kaufmann R, et al.: SIMcheck: a toolbox for successful super-resolution structured illumination microscopy. Sci Rep. 2015; 5: 15915 PubMed Abstract | Publisher Full Text | Free Full Text

35. Brent JR, Werner KM, McCabe BD: Drosophila larval NMJ dissection. J Vis Exp. 2009; (24): 1107.

PubMed Abstract | Publisher Full Text | Free Full Text 


\title{
Open Peer Review
}

\section{Current Peer Review Status:}

Version 1

Reviewer Report 29 September 2021

https://doi.org/10.21956/wellcomeopenres.18310.r45668

(C) 2021 Swedlow J et al. This is an open access peer review report distributed under the terms of the Creative Commons Attribution License, which permits unrestricted use, distribution, and reproduction in any medium, provided the original work is properly cited.

\author{
Jason R Swedlow \\ Centre for Gene Regulation and Expression, School of Life Sciences, University of Dundee, Dundee, \\ UK

\section{Emil Rozbicki} \\ Glencoe Software, Inc, Seattle, WA, USA
}

\section{William Moore}

OME, University of Dundee, Dundee, UK

\section{Sébastien Besson}

Centre for Gene Regulation and Expression, School of Life Sciences, University of Dundee, Dundee, UK

This paper introduces Microscope-Cockpit, a graphical user interface software that has been built on top of the historical software used to control the OMX microscope. It aims to provide a clean and user friendly interface allowing to create complex experimental set-up. Several use cases are included in the article to support the capacity of the software to adapt to different experimental set-ups. Overall the tool described in this article is scientifically valid but we would like to suggest some revisions detailed below.

The Implementation section details the Python requirements of Microscope-Cockpit. Amongst those packages, the Python-microscope library appears as one of the most critical dependencies since it contains all the logic and support for handling and controlling microscopy devices. As part of this review, it came to our attention that this library has been recently published ( https://doi.org/10.1242/jcs.258955) ${ }^{1}$ including a supplementary figure Fig S1 which crossreferences Fig 1 of this paper. The article should thus be amended to cross-reference the Pythonmicroscope publication wherever appropriate.

The article makes no mention of the file format written by Microscope-Cockpit. The Pythonmicroscope paper does not include any detail about the choice of data output for end-users either. From our minimal testing of the application, the only option available to the user is to write data as "DV files (.dv)", a proprietary file format. Is this the only choice available to the user? If so, this feels like a limitation of the current implementation which contradicts the claim for the open and universal mission of Cockpit. We suggest a paragraph should be added to the Implementation 
section that discusses the Data Generation/Output including a description of the file format as well as the tooling available for end-users of the acquisition system to read and analyze imaging data generated by Cockpit.

In the section discussing Cockpit in relationship Micro-Manager, a proposed key strength of Cockpit is its Python implementation allowing the "strict separation of the user interface from the hardware control components" while "the majority of [Micro-Manager] interface must be written in Java; however the system also needs a $\mathrm{C} / \mathrm{C}++$ layer to interface the Java code to $\mathrm{C} / \mathrm{C}++$ based system libraries". This argument omits Pycro-Manager, a Python bridge for Micro-Manager, published earlier this year (https://doi.org/10.1038/s41592-021-01087-6) ${ }^{2}$ and mentioned in the aforementioned Python-microscope publication. The section mentioned above should be amended to cite this paper and include Pycro-Manager in the comparison with Cockpit. Much is made of the ability to use Python for custom modifications and the integration of thirdparty libraries. The text mentions that "the microscope control can easily be modified to make use of the extensive machine learning algorithms available in easy to integrate libraries" but this sentence rather suggests the extensibility is a property of the underlying python-microscope library rather than the Cockpit interface. The Cockpit reference documentation ( https://www.micron.ox.ac.uk/software/cockpit/) primarily describes the installation and the configuration of the software. To support the extensibility claim, we would suggest either to introduce a public example of Python extension to the default Cockpit UI and/or an amendment to the online documentation that would demonstrate this extension which could be linked from the article.

Together with python-microscope paper, this paper introduces a new open-source solution for microscope control and imaging acquisition. Both in the introduction and in the discussion, Cockpit is compared to several equivalent commercial and open-source solutions, including the well-established Micro-Manager. This comparison is important and particularly useful for end users and imaging facility managers who need to make informed decisions e.g. when investing in new equipment or technologies. We suggest to make the outcome of this discussion more explicit and prominent e.g. under a form of a summary table that would include the different available ecosystems (individual hardware solutions, LabView, python-microscope/Microscope-Cockpit and Micro-Manager/Pycro-Manager) and compare their respective advantages and drawbacks.

\section{References}

1. Pinto DMS, Phillips MA, Hall N, Mateos-Langerak J, et al.: Python-microscope: A new open source Python library for the control of microscopes.J Cell Sci. 2021. PubMed Abstract | Publisher Full Text 2. Pinkard H, Stuurman N, Ivanov I, Anthony N, et al.: Pycro-Manager: open-source software for customized and reproducible microscope control. Nature Methods. 2021; 18 (3): 226-228 Publisher Full Text

Is the rationale for developing the new software tool clearly explained? Partly

Is the description of the software tool technically sound? Yes

Are sufficient details of the code, methods and analysis (if applicable) provided to allow replication of the software development and its use by others? 
Is sufficient information provided to allow interpretation of the expected output datasets and any results generated using the tool?

No

\section{Are the conclusions about the tool and its performance adequately supported by the findings presented in the article?}

Yes

Competing Interests: No competing interests were disclosed.

Reviewer Expertise: bioimaging, image informatics, software engineering, data management

We confirm that we have read this submission and believe that we have an appropriate level of expertise to confirm that it is of an acceptable scientific standard, however we have significant reservations, as outlined above.

Author Response 14 Dec 2021

Ian Dobbie, University of Oxford, South Parks Road, Oxford, UK

Response to review from authors.

Authors responses are in italics

New text in the manuscript are in bold

The Implementation section details the Python requirements of Microscope-Cockpit. Amongst those packages, the Python-microscope library appears as one of the most critical dependencies since it contains all the logic and support for handling and controlling microscopy devices. As part of this review, it came to our attention that this library has been recently published (https://doi.org/10.1242/jcs.258955) ${ }^{1}$ including a supplementary figure Fig S1 which cross-references Fig 1 of this paper. The article should thus be amended to cross-reference the Python-microscope publication wherever appropriate.

We thank the reviewers for pointing this out. The Python-Microscope publication has been accepted at JCS since we submitted the article. We have revised the manuscript to reference the published article rather than the biorXiv preprint.

The article makes no mention of the file format written by Microscope-Cockpit. The Pythonmicroscope paper does not include any detail about the choice of data output for end-users either. From our minimal testing of the application, the only option available to the user is to write data as "DV files (.dv)", a proprietary file format. Is this the only choice available to the user? If so, this feels like a limitation of the current implementation which contradicts the claim for the open and universal mission of Cockpit. We suggest a paragraph should be added to the Implementation section that discusses the Data Generation/Output including a description of the file format as well as the tooling available for end-users of the acquisition system to read and analyze imaging data generated by Cockpit. 
We thank the reviewers for raising this point. While it is correct that Microscope-Cockpit only saves images in the '. $d v$ ' file format, it is a slight variant of the open MRC file format commonly used in the electron microscopy community. The MRC format is extensively documented at https://Www.ccpem.ac.uk/mrc_format/mrc2014.php. The sight variations to the encoded metadata reflect differences between the relevant data in optical and electron microscopy. Crucially, there is considerable support within open source software that supports '. $d v$ ' files, including Bio-Formats for importing data into ImageJ, OMERO and Matlab. Therefore, users can easily convert to other formats and use popular programs to analyse the data output by Microscope-Cockpit based microscopes. We have revised the manuscript to address this point and changed the software package documentation to include an explanation of this as well as information about the software that supports the '. $d v^{\prime}$ format, and explicit definition of what metadata we save in the files. We have added a section on output image files which explicitly mentions these points:

\section{Output image files}

Experimental images are saved into files utilising the '.dv' file format, typically multiple wavelengths, Z-slices and time points into a single file. Images from the mosaic window and single snaps can also be saved into this format, with the mosaic saved files having an associated text file defining $X Y Z$ positions of each collected image. The '.dv' format is an extension of the mrc file format, defined in detail in the MRC/CCP4 2014 file format specification. The CCP4 consortium of the EM community continue to support and extend this file format. This support includes file validators and a detailed specification, which is compatible with the files used here but not identical.

The optical microscopy specific metadata are covered in the documentation. Although relatively uncommon, the file format is supported by the Bio-Formats project allowing import of '.dv' files, along with the associated metadata into software using this library including ImageJ, OMERO and Matlab. Additionally, the Chromagnon image alignment tool will read and write '. $d v^{\prime}$ files and it is the native format for DeltaVision microscopes utilising the commercial package SoftWoRx.

In the section discussing Cockpit in relationship Micro-Manager, a proposed key strength of Cockpit is its Python implementation allowing the "strict separation of the user interface from the hardware control components" while "the majority of [Micro-Manager] interface must be written in Java; however the system also needs a $\mathrm{C} / \mathrm{C}++$ layer to interface the Java code to $\mathrm{C} / \mathrm{C}++$ based system libraries". This argument omits Pycro-Manager, a Python bridge for Micro-Manager, published earlier this year (https://doi.org/10.1038/s41592-02101087-6) ${ }^{2}$ and mentioned in the aforementioned Python-microscope publication. The section mentioned above should be amended to cite this paper and include Pycro-Manager in the comparison with Cockpit.

We thank the reviewers for pointing out this paper which was again published since our original submission and we have now added discussion about Pycro-Manager to the paper. We have revised the manuscript by adding the following text to the discussion section: 
It should be noted that Pycro-Manager [pinkard2021] has recently been developed and released to allow much closer integration between Python and uManager. PycroManager seems likely to fill a similar position to Microscope-Cockpit, allowing direct Python based control of imaging experiments, integration with online analysis and a range of other functionality. As this is a recent development, we have not explored this package in any detail.

Much is made of the ability to use Python for custom modifications and the integration of third-party libraries. The text mentions that "the microscope control can easily be modified to make use of the extensive machine learning algorithms available in easy to integrate libraries" but this sentence rather suggests the extensibility is a property of the underlying python-microscope library rather than the Cockpit interface. The Cockpit reference documentation (https://www.micron.ox.ac.uk/software/cockpit/) primarily describes the installation and the configuration of the software. To support the extensibility claim, we would suggest either to introduce a public example of Python extension to the default Cockpit UI and/or an amendment to the online documentation that would demonstrate this extension which could be linked from the article.

The reviewers make a good point that would significantly strengthen the paper. We have written a short script that utilises some of the embedded functionality to find and mark cell nuclei in a typical DAPI stained sample. The code, along with a test image set, config files and detailed instructions on how to set it up are collected into a zenodo repository and available via a doi (10.5281/zenodo.5745648) link. In fact, this simulated microscope approach is a very powerful approach to setting up and testing such scripts. We have also added the following text to the manuscript.

\section{Example simple extension in Python}

We include an example script which demonstrates how the functionality of cockpit can easily be extended in python. The script finds DAPI stained cell nuclei in images, utilising the mosaic functionality to scan large areas and the point marking features to record the centroids of the detected nuclei. The script utilises the OpenCV framework to detect large roughly circular objects in images. Images taken for the mosaic are also trapped by the code which Gaussian blurs to reduce noise, binarise and finally applies a Hough transform to find circular objects. The locations of these objects in the image are then transformed into stage coordinates and added to the marked point list. The Python code, a test image and detailed instructions on how to setup a simulated microscope to run this without any microscope hardware is detailed at https://doi.org/10.5281/zenodo.5745648.

For this simple example the parameters are fixed and tuned to the data set used, and select a subset of nuclei within a size range and with defined circularity. The current code also doesn't reliably detect nuclei at the edges between images in the mosaic scan. However, it can easily detect a large number of cell positions to create a point list which could then be run through a multi-site experiment to collect data such as a 
3D, multi-channel stack, or time lapse on a large number of cells semi-automatically.

Together with python-microscope paper, this paper introduces a new open-source solution for microscope control and imaging acquisition. Both in the introduction and in the discussion, Cockpit is compared to several equivalent commercial and open-source solutions, including the well-established Micro-Manager. This comparison is important and particularly useful for end users and imaging facility managers who need to make informed decisions e.g. when investing in new equipment or technologies. We suggest to make the outcome of this discussion more explicit and prominent e.g. under a form of a summary table that would include the different available ecosystems (individual hardware solutions, LabView, python-microscope/Microscope-Cockpit and Micro-Manager/Pycro-Manager) and compare their respective advantages and drawbacks.

We thank the reviewers for this suggestion and we have added a table of feature comparison between a range of microscope control options as a traffic light colour coded table and the following text to the manuscript.

Table 1 shows a comparison between various features of the previously described microscope control software options. We have ranked features of the control software options with a traffic light colour scheme with green being best and red least good. The table includes the approaches mentioned above along with Matlab, a common alternative to LabVIEW, a more conventional programming package with a range of extensions and with a large support base, and MetaMorph/ SlideBook included as representatives of commercial generalised microscope control packages.

[New table included in revised version]

Table 1: A symbolic representation of the relative strengths of different microscope control approaches in a number of areas with a traffic light colour scheme, with red worst and green best. Separate control programs involve using a separate control program for each piece of hardware. In general, this software is free, however some components such as cameras might require a separate software purchase from the manufacturer. MetaMorph and SlideBook are examples of generalised commercial software designed for microscope control and provided by third parties, these are only two examples of a range of such packages.

Competing Interests: No competing interests were disclosed.

Reviewer Report 14 September 2021

https://doi.org/10.21956/wellcomeopenres.18310.r45664 
(c) 2021 Harrington K. This is an open access peer review report distributed under the terms of the Creative Commons Attribution License, which permits unrestricted use, distribution, and reproduction in any medium, provided the original work is properly cited.

\section{Kyle Harrington}

Max Delbrueck Center for Molecular Medicine, Berlin, Germany

What about the availability of open source drivers through micromanager? Although it is somewhat recognized that the micromanager API/architecture is not ideal, it is certainly recognized that the large number of already existing drivers is a key strength. How is the availability of drivers for Cockpit?

How does Cockpit compare to Pycro-manager (Pycro-Manager: open-source software for customized and reproducible microscope control, Pinkard et al., 2021, Nature Methods), ${ }^{1}$ which enjoys the benefits of the existing micromanager ecosystem while also providing a Python interface?

Overall, Cockpit seems like a strong contribution. I see a lot of potential, but it is not clear that it is quite competitive with micromanager yet (which is fine, that will come with time). Integration with a tool like napari would be a great choice for broadening the usability of Cockpit. It is nice to see that Cockpit is being tested on multiple systems, although as mentioned it would be good to see a fair comparison between Cockpit's current (and future planned) drivers relative to a mature project like micromanager.

\section{References}

1. Pinkard H, Stuurman N, Ivanov I, Anthony N, et al.: Pycro-Manager: open-source software for customized and reproducible microscope control. Nature Methods. 2021; 18 (3): 226-228 Publisher Full Text

Is the rationale for developing the new software tool clearly explained?

Yes

Is the description of the software tool technically sound?

Yes

Are sufficient details of the code, methods and analysis (if applicable) provided to allow replication of the software development and its use by others?

Yes

Is sufficient information provided to allow interpretation of the expected output datasets and any results generated using the tool?

Yes

Are the conclusions about the tool and its performance adequately supported by the findings presented in the article?

Yes 
Competing Interests: No competing interests were disclosed.

Reviewer Expertise: Image analysis, Computer science, Software engineering, Computational biology, Python, Java, C++, Lisp

I confirm that I have read this submission and believe that I have an appropriate level of expertise to confirm that it is of an acceptable scientific standard.

Author Response 14 Dec 2021

Ian Dobbie, University of Oxford, South Parks Road, Oxford, UK

Response to review from Authors.

What about the availability of open source drivers through micromanager? Although it is somewhat recognized that the micromanager API/architecture is not ideal, it is certainly recognized that the large number of already existing drivers is a key strength. How is the availability of drivers for Cockpit?

This is an interesting idea and we have considered it. There are two major arguments against this approach. Firstly this would require importing the whole of the uManager infrastructure, the java JVM etc... Secondly our brief exploration appeared to show that matching devices to the fixed device type specific API in Python-Microscope, the library we use to interface directly to the hardware, would involve substantial effort for each device. We felt it was more productive o apply this effort to implementing the device directly in Python. This probably took roughly the same effort and enabled us to avoid the uManager overhead.

How does Cockpit compare to Pycro-manager (Pycro-Manager: open-source software for customized and reproducible microscope control, Pinkard et al., 2021, Nature Methods), which enjoys the benefits of the existing micromanager ecosystem while also providing a Python interface?

We thank the reviewer for pointing out this paper which was published since our original submission and we have now added discussion about Pycro-Manager to the paper. We have revised the manuscript by adding the following text to the discussion section:

It should be noted that Pycro-Manager [pinkard2021] has recently been developed and released to allow much closer integration between Python and uManager. This seems likely to fill a similar position as Microscope-Cockpit, allowing direct Python based control of imaging experiments, integration with online analysis and a range of other functionality. As this is a recent development we have not explored this package in any detail.

Competing Interests: No competing interests were disclosed. 